OPEN

SUBJECT AREAS:

FIBRE LASERS

ULTRAFAST LASERS

Received

2 August 2013

Accepted

3 September 2013

Published

23 September 2013

Correspondence and requests for materials should be addressed to X.L. lliuxueming72@ yahoo.com)

\title{
Versatile multi-wavelength ultrafast fiber laser mode-locked by carbon nanotubes
}

\author{
Xueming Liu', Dongdong Han' ', Zhipei Sun ${ }^{2,3}$, Chao Zeng' ', Hua Lu', Dong Mao', Yudong Cui' \\ \& Fengqiu Wang ${ }^{2}$
}

\begin{abstract}
${ }^{1}$ State Key Laboratory of Transient Optics and Photonics, Xi'an Institute of Optics and Precision Mechanics, Chinese Academy of Sciences, Xi'an 710119, China, ${ }^{2}$ Department of Engineering, University of Cambridge, $9 \mathrm{JJ}$ Thomson Avenue, Cambridge, CB3 OFA, UK, ${ }^{3}$ Department of Micro- and Nanosciences, Aalto University, PO Box 13500, FI-00076 Aalto, Finland.
\end{abstract}

Multi-wavelength lasers have widespread applications (e.g. fiber telecommunications, pump-probe measurements, terahertz generation). Here, we report a nanotube-mode-locked all-fiber ultrafast oscillator emitting three wavelengths at the central wavelengths of about 1540, 1550, and $1560 \mathrm{~nm}$, which are tunable by stretching fiber Bragg gratings. The output pulse duration is around 6 ps with a spectral width of $\sim 0.5 \mathrm{~nm}$, agreeing well with the numerical simulations. The triple-laser system is controlled precisely and insensitive to environmental perturbations with $<0.04 \%$ amplitude fluctuation. Our method provides a simple, stable, low-cost, multi-wavelength ultrafast-pulsed source for spectroscopy, biomedical research and telecommunications.

U ltrafast mode-locked fiber lasers have widespread applications (e.g. fiber telecommunications, pumpprobe measurements, terahertz generation), due to their various advantages (e.g. small footprint, high stability, efficient heat dissipation, low-cost ${ }^{1-6}$ ). Thus far, single-wavelength ultrafast fiber lasers have been investigated theoretically and demonstrated experimentally ${ }^{7-11}$. However, there are few papers reporting multiwavelength ultrafast fiber lasers ${ }^{12-16}$, which mainly use the naturally formed birefringence of single-mode fiber (SMF) for lasing wavelength selection. In such fiber lasers, the output wavelengths cannot be controlled precisely due to the difficulty of accurately adjusting the birefringence in SMF. Therefore, these multi-wavelength pulsed fiber lasers are not very stable, and output central wavelengths are not selectable to meet the requirements of various applications. To address this issue, a simple way is to use fiber Bragg gratings for the wavelength selection, which can offer all-fiber based alignment-free structure ${ }^{17-19}$. Furthermore, fast development in the chirped fiber Bragg grating (CFBG) fabrication technology has been achieved to provide changeable dispersion, broad bandwidth, and tunable transmittance wavelength covering all major laser wavelengths (e.g. 1, 1.55, and $2 \mu \mathrm{m}$ ). These make CFBG an ideal wavelength selection component for ultrafast broadband fiber lasers.

To achieve multi-wavelength pulsed lasers, another key element is the saturable absorber, which can operate at multi-wavelengths (i.e. broad operation bandwidth ${ }^{20}$ ). Currently, various saturable absorbers, such as nonlinear loop mirrors ${ }^{21,22}$, nonlinear polarization rotation ${ }^{23,24}$, semiconductor saturable absorber mirrors (SESAMs) ${ }^{20,25,26}$, carbon nanotubes ${ }^{7,10,11,27-30}$, and graphene ${ }^{8,31-34}$, have been employed for ultrafast pulse generation. Among these saturable absorbers, nanotube and graphene are particularly interesting for multi-wavelength pulsed lasers as they both exhibit extraordinarily broad operation bandwidth for multi-wavelength pulse generation. Indeed, such unique broadband property has been experimentally confirmed for nanotube ${ }^{29,35}$ and graphene ${ }^{33}$ by wavelength tunable $^{32,35}$ and dual-wavelength ${ }^{30,34}$ pulsed lasers. However, triple-wavelength mode-locking laser has not been demonstrated with carbon nanotubes.

In this article, we report a compact nanotube-mode-locked all-fiber laser system based on CFBGs, delivering three lasing wavelengths simultaneously. The output central wavelengths are 1539.5, 1549.5, and $1559.5 \mathrm{~nm}$, respectively, which can be accurately selected by CFBGs. The output wavelengths are tunable by stretching CFBGs. The pulse durations of three wavelengths are 6.3, 6.7, and 5.9 ps, respectively. Our laser is insensitive to environmental perturbation with near-transform-limited pulses, and thus is viable for various practical applications, such as fiber telecommunications, pump-probe measurements, and terahertz generation. The proposed method of precisely selecting output wavelengths by fiber gratings and using nanotubes with broad operation bandwidth can be readily adopted for other fiber lasers from 1 to $2 \mu \mathrm{m}$. 


\section{Results}

Nanotube-based compact all-fiber triple-wavelength laser system. The schematic diagram of our nanotube-based compact all-fiber triple-laser system is shown in Fig. 1(a). The laser system consists of a wavelength-division multiplexer (WDM), a fused coupler with $10 \%$ output ratio, two polarization controllers (PCs), three CFBGs, a 5 -m-long erbium-doped fiber (EDF) with $6 \mathrm{~dB} / \mathrm{m}$ absorption at $980 \mathrm{~nm}$, a single-wall carbon nanotube (SWNT) saturable absorber, and a circulator. The EDF and SMF have dispersion parameters of about 11.6 and $-22 \mathrm{ps}^{2} / \mathrm{km}$ at $1550 \mathrm{~nm}$, respectively. CFBGs, written on a standard SMF, have a super-Gaussian reflection profile with a bandwidth of $\sim 1 \mathrm{~nm}$ (Fig. 1(b)). The dispersion parameter of these three CFBGs is $\sim 2.2 \mathrm{ps}^{2} / \mathrm{cm}$ with the length of $\sim 10 \mathrm{~mm}$, and the corresponding central transmittance wavelengths $\lambda_{1-3}$ are 1539.5, 1549.5 , and $1559.5 \mathrm{~nm}$, respectively. The wavelengths of our laser
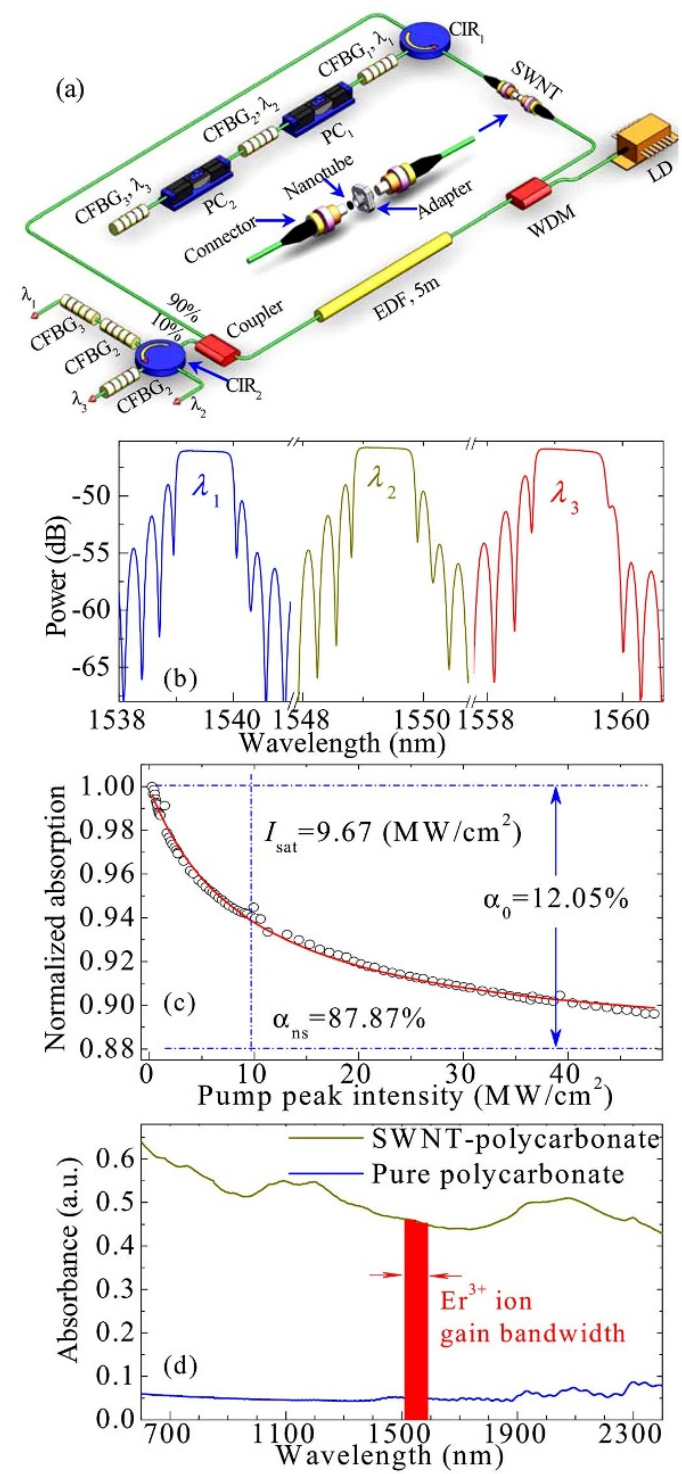

Figure $1 \mid$ (a) Laser setup. Inset: the assembly of SWNT saturable absorber. EDF, erbium-doped fiber; WDM, wavelength-division multiplexer; PC, polarization controller; LD, laser diode; CIR, circulator; CFBG, chirped fiber Bragg grating; SWNT, single wall carbon nanotube. (b) Reflection spectra of three $\mathrm{CFBG}_{1-3}$. (c) Nonlinear absorption characterization of the SWNT saturable absorber. The solid curve is fitted from the experimental data (circle symbols). (d) Absorption spectrum of the SWNTpolycarbonate composite and pure polycarbonate. The red stripe illustrates the spectral gain region of the $\mathrm{Er}^{3+}$-doped fiber. output are separated by another three CFBGs for characterization of individual wavelengths.

The integrated SWNT-based fiber device is realized by sandwiching a $\sim 2 \mathrm{~mm}^{2}$ sample between two fiber connectors (see Methods), as shown in inset of Fig. 1(a). The normalized nonlinear absorption of our integrated SWNT absorber is experimentally measured with a homemade ultrafast laser at $1550 \mathrm{~nm}$, as shown in Fig. 1(c). According to a simplified two-level saturable absorber model ${ }^{35,36}$, the experimental data are fitted as the solid curve of Fig. 1(c). Figure 1(d) shows the absorption spectrum of the SWNT-polycarbonate composite in comparison with pure polycarbonate, which is measured by a spectrometer (JASCO V-570 UV-vis-NIR).

Experimental observations. Continuous wave (CW) operation starts at the pump power of $P \approx 9 \mathrm{~mW}$, and self-starting mode-locking is observed at $P \approx 15 \mathrm{~mW}$. With the appropriate setting of two PCs, output at three wavelengths is generated from the oscillator. The typical output spectra of three lasers $\lambda_{1-3}$ at $P \approx 45 \mathrm{~mW}$ are shown in Figs. 2(a), 2(c), and 2(e) with the central wavelengths at $1539.5,1549.5$, and $1559.5 \mathrm{~nm}$, respectively. The corresponding autocorrelation traces of the experimental data and the $\mathrm{sech}^{2}-$ shaped fit for $\lambda_{1-3}$ are shown in Figs. 2(b), 2(d), and 2(f). It is found that the optical spectra have sidebands, which are the typically spectral characteristics of standard solitons $s^{1,37,38}$. The full width at half maximum (FWHM) spectral widths at different wavelengths are about $0.47,0.41$, and $0.49 \mathrm{~nm}$, respectively. The output pulse durations $(\Delta \tau)$ are about 6.3, 6.7, and 5.9 ps. The calculated time-bandwidth products at three different wavelengths are about $0.37,0.35$, and 0.36 , respectively, which are slightly larger than the value of 0.315 for the transform-limited sech $^{2}$-shaped pulses. It is worth noting that here the output spectral width and pulse duration are limited by the bandwidth (i.e. $1 \mathrm{~nm}$ ) of CFBGs used in the cavity. Broader bandwidth CFBGs in principle can offer broader spectral width, and thus shorter pulse duration.

Figures 3(a-d) show the RF spectra and oscilloscope traces of lasers, respectively. Figures $3(\mathrm{a}-\mathrm{b})$ are the fundamental RF spectra with the $1 \mathrm{~Hz}$ resolution and the $100 \mathrm{~Hz}$ span for three lasers $\lambda_{1-3}$.
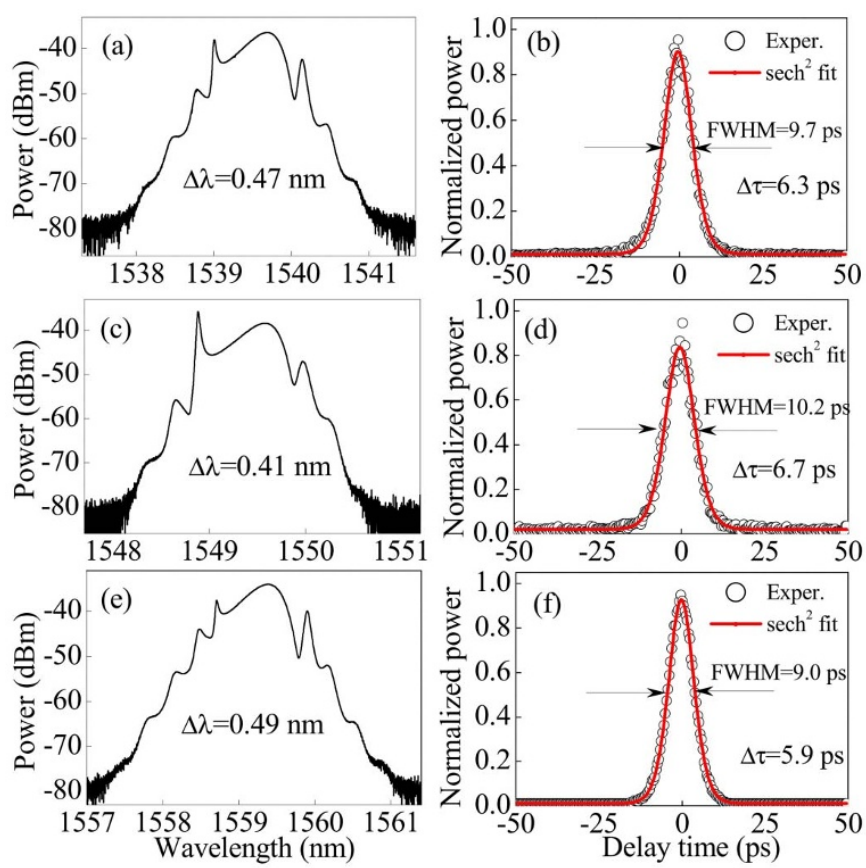

Figure $2 \mid$ Optical spectra of the experimental observations for three lasers (a) $\lambda_{1}$, (c) $\lambda_{2}$, and (e) $\lambda_{3}$. Autocorrelation traces of the experimental data (circle symbols) and sech $^{2}$-shaped fit (solid curves) for (b) $\lambda_{1}$, (d) $\lambda_{2}$, and (f) $\lambda_{3}$. 

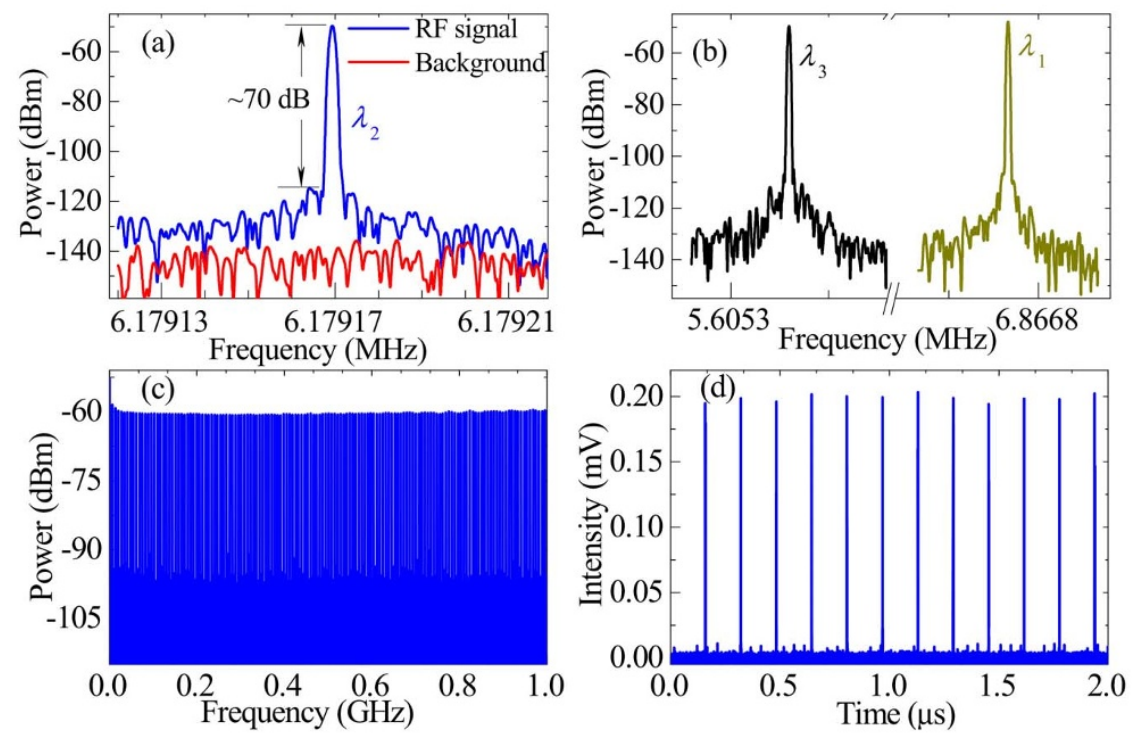

Figure 3 Typically experimental results: the fundamental RF spectra with the resolution of $1 \mathrm{~Hz}$ and the span of $100 \mathrm{~Hz}$ for three lasers (a) $\lambda_{2}$ and (b) $\lambda_{1}$ and $\lambda_{3}$. The fundamental repetition rates of $\lambda_{1-3}$ are $6.86678,6.17917$, and $5.60533 \mathrm{MHz}$, respectively. (c) Wideband RF spectrum up to $1 \mathrm{GHz}$ and (d) oscilloscope traces up to $2 \mu$ s for the laser $\lambda_{2}$.

Figures 3(c) and 3(d) are the wideband RF spectrum up to $1 \mathrm{GHz}$ and the oscilloscope traces up to $2 \mu$ s for the laser $\lambda_{2}$, respectively. Figure 3(a) exhibits that the repetition rate of the fundamental harmonic frequency is $6.17917 \mathrm{MHz}$, corresponding to $\sim 161.8 \mathrm{~ns}$ round-trip time, as shown in Fig. 3(d). No spectrum modulation is observed over $1 \mathrm{GHz}$ (Fig. 3(c)), indicating no Q-switching instabilities.

The RF spectrum in Fig. 3(a) gives a signal-to-noise ratio $\sim 70 \mathrm{~dB}$ ( $10^{7}$ contrast), showing low-amplitude fluctuations and good modelocking stability ${ }^{10,39}$. With the power ratio of $\Delta P \approx 10^{-7}$, the frequency resolution $\Delta f_{\text {res }}=1 \mathrm{~Hz}$, and the frequency width (FWHM) of $\Delta f_{A} \approx 1.3 \mathrm{~Hz}$ (Fig. 3(a)), we estimate an amplitude fluctuation $\Delta E$ / $E \approx 3.6 \times 10^{-4}$ from the equation $\Delta E / E=\left(\Delta P \Delta f_{A} / \Delta f_{\text {res }}\right)^{1 / 2}{ }^{39}$. Note that no pulse is observed in the experimental observations if SWNT is removed.

Numerical results. The typical results of numerical simulations for three lasers $\lambda_{1-3}$ in the mode-locking regime are demonstrated in Fig. 4. Parameters are chosen to match the experimental values (see Methods). We can see from Fig. 4 that the spectral width and pulse duration of $\lambda_{1-3}$ are $0.474,0.401,0.486 \mathrm{~nm}$, and $6.39,6.79,6.01 \mathrm{ps}$, respectively. So the time-bandwidth products of $\lambda_{1-3}$ are about 0.38 , 0.34 , and 0.36, respectively, showing that they are $\operatorname{sech}^{2}$-shaped pulses rather than Gaussian-shaped pulses. The numerical results (Fig. 4) are in good agreement with the experimental observations, as shown in Fig. 2. Figure 5 shows the evolution of pulse along the oscillator for the laser $\lambda_{2}$. Obviously, it is dynamic rather than static. The evolution of pulse profile in a round trip is demonstrated in the supplemental material.

\section{Discussion}

In the experiments, the proposed oscillator (Fig. 1) fails to simultaneously delivering three wavelengths if SWNT is replaced by such saturable absorbers as nonlinear loop mirror, nonlinear polarization rotation, SESAM, and graphene. It attributes to the inherent characteristic of SWNT, which has highly environmental stability and is independent of the polarization of pulses evolving in the laser cavity $^{10,35,40,41}$.

Based on the cascade of CFBGs, the laser system with more than three wavelengths (e.g. four and five wavelengths) can be achieved in principle. By stretching CFBGs in our experiments, its central wavelength is tunable. Figure 6 demonstrates the output spectra at ten wavelengths within the tuning range from $\sim 1560$ to $1565 \mathrm{~nm}$. The experimental results show that the spectral width and pulse duration are almost unchanged, indicating the stability of our output pulses. Note that other two wavelengths also can be tuned but with limited wavelength range. For example, the $\lambda_{1}$ can be tuned from 1540 to $1546 \mathrm{~nm}$, and the $\lambda_{2}$ can be tuned from 1550 to $1556 \mathrm{~nm}$. The tuning range is determined by the CFBGs.

\section{Methods}

SWNT film. SWNT with the tube diameter $<2 \mathrm{~nm}$ is grown with the catalytic chemical vapor decomposition method using $\mathrm{CH}_{4}$ as the carbon source and Co as the catalyst. $0.5 \mathrm{mg} \cdot \mathrm{mL}^{-1} \mathrm{SWNT}$ solution is prepared by dispersing SWNT powder in de-ionized water with sodium dodecyl benzenesulfonate (SDBS) using a sonicator
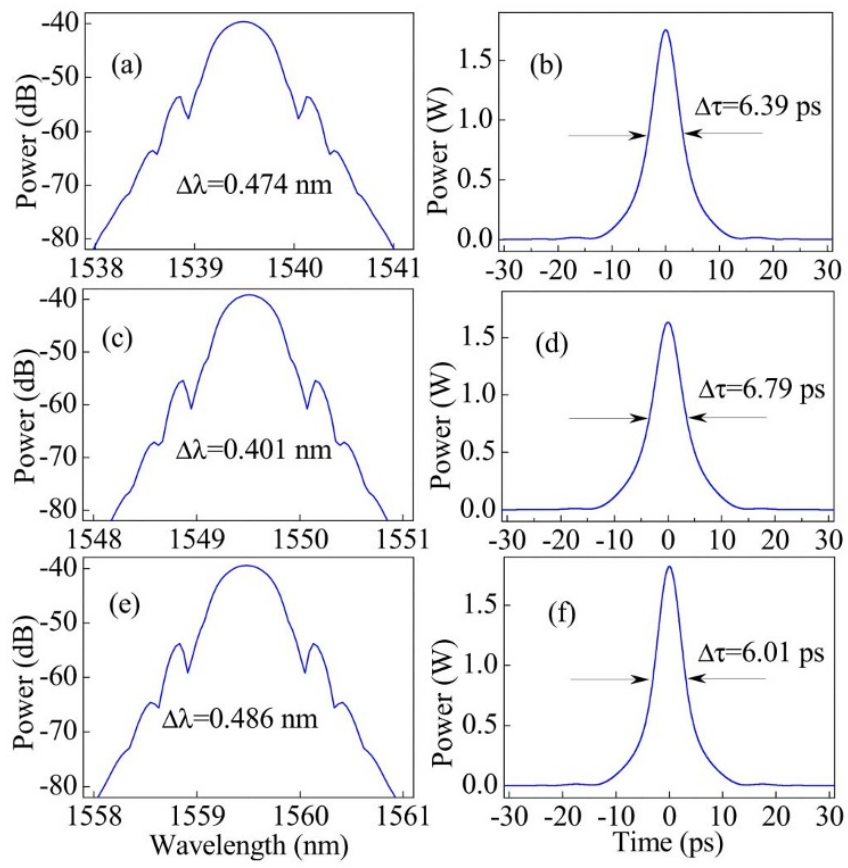

Figure $4 \mid$ Optical spectra and pulse profiles of the numerical simulations for three lasers (a) and (b) $\lambda_{1}$, (c) and (d) $\lambda_{2}$, (e) and (f) $\lambda_{3}$. 


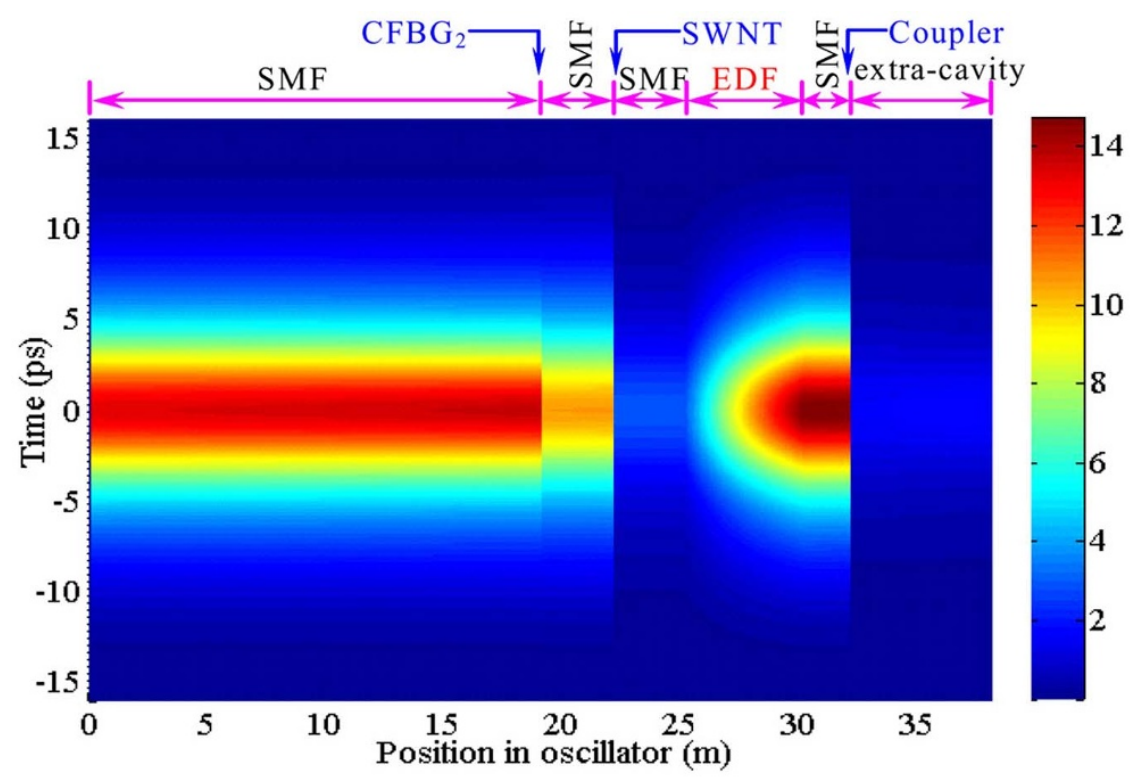

Figure $5 \mid$ Pulse evolution of laser $\lambda_{2}$ along the intra- and extra-cavity position. The supplemental material demonstrates the evolution of pulse profile along the oscillator in detail.

system operating at $20 \mathrm{kHz}$ with $180 \mathrm{~W}$ power for 5 hours. To avoid unwanted scattering losses from aggregates and bubbles, the resulting dispersion is centrifuged at $12000 \mathrm{~g}$ for an hour, and the upper $90 \%$ of the supernatant is then collected. $10 \mathrm{wt} \%$ aqueous polyvinyl alcohol (PVA) solution and $0.5 \mathrm{mg} \cdot \mathrm{mL}^{-1}$ SWNT solution are mixed at the volume ratio of $1: 2$ overnight by a magnetic stirrer. Slow evaporation under ambient temperature and pressure results in a $\sim 30-\mu \mathrm{m}$-thick freestanding SWNT-PVA composite film.

Measurement method. An optical spectrum analyzer (Yokogawa AQ-6370), an autocorrelator, a 6-GHz oscilloscope, a radio-frequency (RF) analyzer, and a $10-\mathrm{GHz}$ photodetector are used to measure the laser output performances.

Numerical simulation. To confirm the experimental observations, we numerically simulate the pulse formation at three wavelengths in the oscillator. The modeling includes such the physics terms as the group velocity dispersion of fiber, the self-phase modulation, the dispersion of CFBGs, and the saturated gain with a finite bandwidth. Thus the extended nonlinear Schrödinger equation is used to describe the pulse propagation in the laser oscillator ${ }^{42}$,

$$
\frac{\partial A}{\partial z}+i \frac{\beta_{2}}{2} \frac{\partial^{2} A}{\partial t^{2}}=g / 2 A+i \gamma|A|^{2} A+\frac{g}{2 \Omega_{g}^{2}} \frac{\partial^{2} A}{\partial t^{2}} .
$$

Here $A, \beta_{2}$, and $\gamma$ denote the electric filed envelop of the pulse, the fiber dispersion, and the cubic refractive nonlinearity of the fiber, respectively. The variables $t$ and $z$ represent the time and the propagation distance, respectively. $\Omega_{g}$ is the bandwidth of the gain spectrum. $g$ describes the gain function for the EDF and is expressed by ${ }^{43}$

$$
g=g_{0} / \exp \left(-E_{p} / E_{s}\right)
$$

where $g_{0}, E_{p}$, and $E_{s}$ are the small-signal gain coefficient related to the doping concentration, the pulse energy, and gain saturation energy that relies on pump

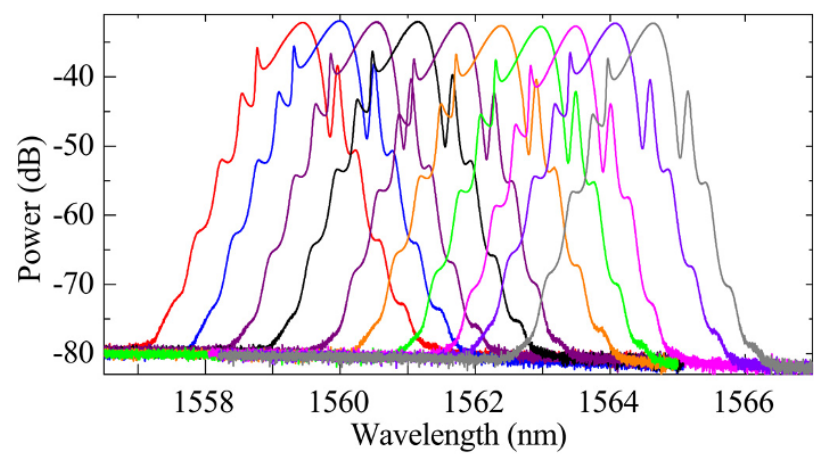

Figure 6 | Output spectra at ten different wavelengths by stretching $\mathrm{CFBG}_{3}$ in the cavity. power, respectively. The normalized absorption is fitted according to a simple twolevel saturable absorber model $^{35,36}$

$$
\alpha(I)=\alpha_{\mathrm{ns}}+\alpha_{0} /\left(1+I / I_{\mathrm{sat}}\right) .
$$

Here $\alpha(I)$ is the intensity-dependent absorption coefficient, and $\alpha_{0}, \alpha_{\mathrm{ns}}$ and $I_{\text {sat }}$ are the linear limit of saturable absorption, nonsaturable absorption, and saturation intensity, respectively.

Eq. (1) is solved with a predictor-corrector split-step Fourier method ${ }^{44}$. To numerically simulate the feature and behavior of this laser system, the simulation has started from an arbitrary signal and converged into a stable solution after approximately 200 round trips. In the simulation, we use the following parameters to match the experimental conditions: $g_{0}=6 \mathrm{~dB} / \mathrm{m}, \Omega_{\mathrm{g}}=25 \mathrm{~nm}, E_{s}=55 \mathrm{pJ}, \gamma=$ $4.5 \mathrm{~W}^{-1} \mathrm{~km}^{-1}$ for EDF, $\gamma=1.3 \mathrm{~W}^{-1} \mathrm{~km}^{-1}$ for SMF. The parameters for SWNT saturable absorber are set with the values measured (Fig. 1 (c)), i.e. $\alpha_{0}=12.05 \%, \alpha_{\mathrm{ns}}=$ $87.87 \%$, and $I_{\text {sat }}=9.67 \mathrm{MW} / \mathrm{cm}^{2}$.

1. Liu, X. M. Soliton formation and evolution in passively-mode-locked lasers with ultralong anomalous-dispersion fibers. Phys. Rev. A 84, 023835 (2011).

2. Grelu, P. \& Akhmediev, N. Dissipative solitons for mode-locked lasers. Nat. Photon. 6, 84-92 (2012).

3. Brabec, T. \& Krausz, F. Intense few-cycle laser fields: Frontiers of nonlinear optics. Rev. Mod. Phys. 72, 545-591 (2000).

4. Steinmeyer, G., Sutter, D. H., Gallmann, L., Matuschek, N. \& Keller, U. Frontiers in ultrashort pulse generation: pushing the limits in linear and nonlinear optics. Science 286, 1507-1512 (1999).

5. Sobon, G., Sotor, J. \& Abramski, K. M. Passive harmonic mode-locking in Erdoped fiber laser based on graphene saturable absorber with repetition rates scalable to $2.22 \mathrm{GHz}$. Appl. Phys. Lett. 100, 161109 (2012).

6. Fermann, M. E. \& Hartl, I. Ultrafast fiber laser technol. IEEE J. Sel. Top. Quant. Electron. 15, 191-206 (2009).

7. Solodyankin, M. A. et al. Mode-locked $1.93 \mu \mathrm{m}$ thulium fiber laser with a carbon nanotube absorber. Opt. Lett. 33, 1336-1338 (2008).

8. Bonaccorso, F., Sun, Z., Hasan, T. \& Ferrari, A. C. Graphene photonics and optoelectronics. Nat. Photon. 4, 611-622 (2010).

9. Liu, X. Interaction and motion of solitons in passively-mode-locked fiber lasers. Phys. Rev. A 84, 053828 (2011).

10. Sun, Z. P. et al. Ultrafast Stretched-Pulse Fiber Laser Mode-Locked by Carbon Nanotubes. Nano Res. 3, 404-411 (2010).

11. Kieu, K. \& Wise, F. W. All-fiber normal-dispersion femtosecond laser. Opt. Express 16, 11453-11458 (2008).

12. Li, S., Chan, K. T., Liu, Y., Zhang, L. \& Bennion, I. Multiwavelength picosecond pulses generated form a self-seeded Fabry-Pérot laser diode with a fiber external cavity using fiber Bragg gratings. IEEE Photon. Technol. Lett. 10, 1712-1714 (1998).

13. Yun, L. et al. Observation of dual-wavelength dissipative solitons in a figure-eight erbium- doped fiber laser. Opt. Express 20, 20992-20997 (2012).

14. Zhang, H., Tang, D. Y., Wu, X. \& Zhao, L. M. Multi-wavelength dissipative soliton operation of an erbium doped fiber laser. Opt. Express 17, 12692-12697 (2009). 
15. Mao, D. et al. Dual-wavelength step-like pulses in an ultra-large negativedispersion fiber laser. Opt. Express 19, 3996-4001 (2011).

16. Zhang, Z. X., Xu, Z. W. \& Zhang, L. Tunable and switchable dual-wavelength dissipative soliton generation in an all-normal-dispersion Yb-doped fiber laser with birefringence fiber filter. Opt. Express 20, 26736-26742 (2012).

17. Kashyap, R. Fiber Bragg Gratings, Academic Press, San Diego (1999).

18. Canning, J. Fibre gratings and devices for sensors and lasers. Laser Photon. Rev. 2 , 275-289 (2008).

19. Giles, C. R. Lightwave applications of fiber Bragg gratings. J. Lightwave Technol. 15, 1391-1404 (1997).

20. Keller, U. Recent developments in compact ultrafast lasers. Nature 424, 831-838 (2003)

21. Yun, L. et al. Generation and propagation of bound-state pulses in a passively mode-locked figure-eight laser. IEEE Photon. J. 4, 512-519 (2012).

22. Salhi, M., Haboucha, A., Leblond, H. \& Sanchez, F. Theoretical study of figureeight all-fiber laser. Phys. Rev. A 77, 033828 (2008)

23. Oktem, B., Ulgudur, C. \& Ilday, F. Soliton-similariton fibre laser. Nat. Photon. 4, 307-311 (2010).

24. Komarov, A., Leblond, H. \& Sanchez, F. Theoretical analysis of the operating regime of a passively-mode-locked fiber laser through nonlinear polarization rotation. Phys. Rev. A 72, 063811 (2005).

25. Okhotnikov, O., Grudinin, A. \& Pessa, M. Ultra-fast fibre laser systems based on SESAM technology: new horizons and applications. New J. Phys. 6, 177 (2004)

26. Mao, D. et al. Observation of pulse trapping in a near-zero dispersion regime. Opt. Lett. 37, 2619-2621 (2012).

27. Set, S. Y., Yaguchi, H., Tanaka, Y. \& Jablonski, M. Ultrafast fiber pulsed lasers incorporating carbon nanotubes. IEEE J. Sel. Top. Quant. Electron. 10, 137-146 (2004).

28. Song, Y. W., Yamashita, S. \& Maruyama, S. Single-walled carbon nanotubes for high-energy optical pulse formation. Appl. Phys. Lett. 92, 021115 (2008).

29. Kivisto, S. et al. Carbon nanotube films for ultrafast broadband technology. Opt. Express 17, 2358-2363 (2009)

30. Song, Y. W., Set, S. Y., Yamashita, S., Goh, C. S. \& Kotake, T. 1300-nm pulsed fiber lasers mode-locked by purified carbon nanotubes. IEEE Photon. Technol. Lett. 17, 1623-1625 (2005).

31. Sun, Z. et al. Graphene mode-locked ultrafast laser. ACS Nano 4, 803-810 (2010)

32. He, X. Y., Liu, Z. B. \& Wang, D. N. Wavelength-tunable, passively mode-locked fiber laser based on graphene and chirped fiber Bragg grating. Opt. Lett. 37, 2394-2396 (2012).

33. Sun, Z. et al. A. A stable, wideband tunable, near transform-limited, graphenemode-locked, ultrafast laser. Nano Res. 3, 653-660 (2010).

34. Luo, Z. et al. Graphene-based passively Q-switched dual-wavelength erbiumdoped fiber laser. Opt. Lett. 35, 3709-3711 (2010).

35. Wang, F. et al. Wideband-tuneable, nanotube mode-locked, fibre laser. Nat. Nanotechnol. 3, 738-742 (2008).

36. Garmire, E. Resonant optical nonlinearities in semiconductors. IEEE J. Sel. Top. Quant. Electron. 6, 1094-1110 (2000).

37. Nelson, L. E., Jones, D. J., Tamura, K., Haus, H. A. \& Ippen, E. P. Ultrashort-pulse fiber ring lasers. Appl. Phys. B 65, 277-294 (1997).
38. Liu, X. M. Coexistence of strong and weak pulses in a fiber laser with largely anomalous dispersion. Opt. Express 19, 5874-5887 (2011).

39 . Von der Linde, D. Characterization of the noise in continuously operating modelocked lasers. Appl. Phys. B 39, 201-217 (1986).

40. Mou, C. B., Rozhin, A. G., Arif, R., Zhou, K. M. \& Turitsyn, S. Polarization insensitive in-fiber mode-locker based on carbon nanotube with N-methyl-2 pryrrolidone solvent filled fiber microchamber. Appl. Phys. Lett. 100, 101110 (2012).

41. Chu, S. et al. Ultrafast saturable absorption devices incorporating efficiently electrosprayed carbon nanotubes. Appl. Phys. Lett. 96, 051111 (2010).

42. Liu, X. M. Numerical and experimental investigation of dissipative solitons in passively mode-locked fiber lasers with large net-normal-dispersion and high nonlinearity. Opt. Express 17, 22401-22416 (2009).

43. Agrawal, G. P. Amplification of ultrashort solitons in erbium-doped fiber amplifiers. IEEE Photon. Technol. Lett. 2, 875-877 (1990).

44. Liu, X. \& Lee, B. A fast method for nonlinear Schrödinger equation. IEEE Photon. Technol. Lett. 15, 1549-1551 (2003).

\section{Acknowledgments}

This work was supported by the National Natural Science Foundation of China under Grants 10874239, 61223007, and 11204368.

\section{Author contributions}

X.L. proposed the laser system, completed the numerical simulation, and wrote the main manuscript text. D.H. performed the main experimental results and discussed the numerical simulation. Z.S. discussed the design of the system and considerably improved the manuscript presentation. C.Z. performed the sample preparation of carbon nanotubes H.L. carried out the data analysis and performed the video. D.M. and Y.C. provided technical support and prepared part figures. F.W. contributed to the scientific discussion and discussed the nanotube sample. All authors discussed the results and substantially contributed to the manuscript.

\section{Additional information}

Supplementary information accompanies this paper at http://www.nature.com/ scientificreports

Competing financial interests: The authors declare no competing financial interests.

How to cite this article: Liu, X. et al. Versatile multi-wavelength ultrafast fiber laser mode-locked by carbon nanotubes. Sci. Rep. 3, 2718; DOI:10.1038/srep02718 (2013).

(c) (i) (-) $€$ This work is licensed under a Creative Commons Attribution-

visit http://creativecommons.org/licenses/by-nc-nd/3.0 


\section{SCIENTIFIC REP RTS}

\section{Corrigendum: Versatile multi- wavelength ultrafast fiber laser mode-locked by carbon nanotubes}

Xueming Liu, Dongdong Han, Zhipei Sun, Chao Zeng, Hua Lu, Dong Mao, Yudong Cui \& Fengqiu Wang

Scientific Reports 3:2718; doi: 10.1038/srep02718; published online 23 September 2013; updated on 20 July 2015

This Article contains typographical errors.

In the Results section under subheading 'Nanotube-based compact all-fiber triple-wavelength laser system, "The dispersion parameter of these three CFBGs is $\sim 2.2 \mathrm{ps}^{2} / \mathrm{cm}$ with the length of $\sim 10 \mathrm{~mm}$ " should read: "The dispersion parameter of these three CFBGs is $-2.2 \mathrm{ps}^{2} / \mathrm{cm}$ with the length of $\sim 10 \mathrm{~mm}$ ".

In addition, "Fig. 1(d) shows the absorption spectrum of the SWNT-polycarbonate composite in comparison with pure polycarbonate" should read: "Fig. 1(d) shows the absorption spectrum of the SWNTPVA composite in comparison with pure PVA".

In the caption of Fig. 1(d), "Absorption spectrum of the SWNT-polycarbonate composite and pure polycarbonate" should read: "Absorption spectrum of the SWNT-PVA composite and pure PVA". The figure legends in Fig. 1(d) "SWNT-polycarbonate" and "Pure polycarbonate" should read "SWNT-PVA" and "Pure PVA" respectively. The correct Fig. 1(d) appears below as Fig. 1.

Lastly, in the Methods section under subheading 'Numerical simulation', Equation (2) " $\mathrm{g}=\mathrm{g}_{\mathrm{o}} / \exp \left(-\mathrm{E}_{\mathrm{p}} / \mathrm{E}_{\mathrm{s}}\right)$ " should read: " $\mathrm{g}=\mathrm{g}_{\mathrm{o}}{ }^{*} \exp \left(-\mathrm{E}_{\mathrm{p}} / \mathrm{E}_{\mathrm{s}}\right)$ "

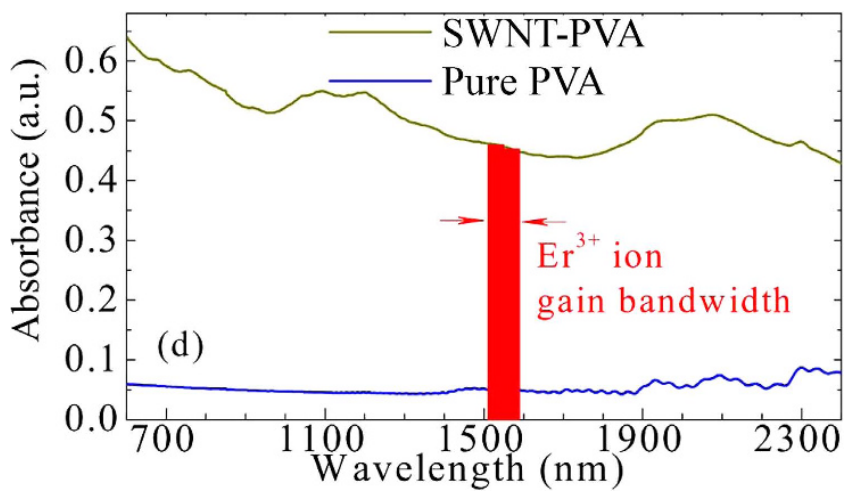

Figure 1. 